\title{
The Radio Shack color computer in the experimental psychology laboratory: An evaluation
}

\author{
A. V. REED \\ Bell Laboratories, Holmdel, New Jersey 07733
}

\begin{abstract}
The Radio Shack color computer is examined from the viewpoint of capabilities needed to program, time, control, and acquire data from typical cognitive psychology experiments. Its capabilities are compared with those of the alternative inexpensive microcomputers discussed in an earlier article (Reed, 1980). The paper also includes a 6809 assembly language routine that permits the measurement of reaction times in video horizontal scan (HS = 4/63 msec) units without hardware modifications and an RSCC BASIC program for unbiased conversion of HS units to milliseconds.
\end{abstract}

As recently as a year ago, the least expensive microcomputer system capable of running most cognitive psychology experiments cost about $\$ 1,400$. While the same system would still cost about $\$ 1,100$ or so today, recent technological developments, such as single-chip display control and memory management circuits, have given us a new generation of much cheaper but nevertheless quite capable small computers. These new machines-the Atari 400, the Commodore VIC, and the Radio Shack color computer-appear to have been designed primarily for home entertainment rather than hobbyist experimentation, and so they do not have the sophisticated hardware expansion capabilities of the older hobby computers. They do, however, have the advantages of good display capabilities and much lower cost. Since many psychology students already have access to cassette tape recorders and standard television sets, the effective cost to a student is just the cost of the computer unit. Recent issues of Byte have carried adver. tisements for Radio Shack color computers for as little as $\$ 310$, with random-access memory expansion to $16 \mathrm{~KB}$ available for an additional $\$ 16$. The total cost is comparable to that of a set of textbooks for a sequence of graduate-level courses. I decided to check out the laboratory potential of the new machines and to see whether it was possible, at long last, to give each student a portable experimental laboratory of her or his own.

Of the three machines listed above, the Atari 400 appears to have the most powerful display generator in its hardware. Unfortunately, its keyboard provides no tactile feedback, which makes it unsuitable for measurement of reaction times. The Commodore VIC appears to have the most limited display capability of the three. While technical specifications of its graphics capabilities were not available as of November 1981, the VIC's text display is limited to 22 characters/row, considerably less than the other two machines. It is equipped with a typewriter-like smooth travel keyboard, which makes it only marginally suitable for reaction time data acquisition applications. Among the three, the Radio Shack color computer comes closest to meeting the "portable experimental psychology laboratory" requirements. Its keyboard gives crisp, snappy tactile feedback, as good as that of most response keys used in traditional psychology laboratories. The display can generate a matrix of up to $256 \times 192$ picture elements, so that visual stimuli should approach the quality obtainable with an Apple II ( $280 \times 192$ pixels). The rest of this paper examines the capabilities of the color computer in greater detail.

\section{PROCESSOR}

The Radio Shack color computer uses a Motorola 6809 (specifically, 6809E) microprocessor. While this processor uses the same external bus as the older 6800 and 6502 , its internal organization incorporates several improvements that make it much easier to use for laboratory applications than its predecessor. First, its machine language is inherently relocatable, so that programs may be written and assembled without the user's having to know, or care, where in the computer's memory each program will reside during execution. Second, the entire "context," that is, the state of the processor and of all its registers (with the obvious exception of the pointer to the stack being used), can be saved (or later recovered) with a single instruction. This is automatically done by the processor for IRQ- and NMI-generated interrupts, so that the user is free from having to worry about which registers get altered during interrupt handlers and subroutines. Third, the instruction set is exceptionally clean and modular. Only 44 instruction types need to be remembered by any assem. bly language programmer, together with 10 register names, eight testable conditions, and 24 addressing modes. All the logically conceivable combinations of 
instructions and addressing modes are available in the instruction set, so there is no need to remember which are available with which, as was the case on the 6502 . Together, these features make assembly language programming, often unavoidable when precise timing is required, easy enough for just about any psychology student.

The instruction set includes several commands not available on earlier processors, three of which are worth mentioning. CWAI (clear condition codes and wait for interrupt) and SYNC (synchronize with masked interrupt input) make it possible to synchronize the processor to an external input with single-cycle precision. On the 6502 , the tightest synchronization loop available could only synchronize processing within six cycles of an external signal. LEA (load effective address) facilitates the handling of arbitrarily dimensioned arrays without explicit arithmetic manipulation of address variables. Since data obtained in psychological experiments frequently have the form of arrays, this capability is an obvious advantage. So is the availability of autoincrement and autodecrement addressing modes, analogous to the corresponding modes in $\mathrm{C}$, at the machine instruction level. Other features, such as the availability of up to four stacks in hardware, are less directly relevant to experimental psychology applications, but they do make it fairly likely that 6809 versions of high-level control application languages, such as $\mathrm{C}$ or FORTH, will be fast enough to use in experiment control when available.

\section{VIDEO DISPLAY}

The color computer uses a VDG (video display generator) integrated circuit (IC) in conjunction with the SAM (synchronous address multiplexer) IC to generate video displays from random-access memory. The computer provides a variety of text and graphic displays ranging from uppercase-only text ( 512 bytes/screen) to $256 \times 192$ pixel graphics $(6 \mathrm{~KB} / \mathrm{screen})$. Unlike the computers discussed in Reed (1980), the color computer's display may originate at any 500 -byte boundary in memory. With $16 \mathrm{~KB}$ of memory, the user can reserve up to $12.5 \mathrm{~KB}$ for video displays: enough for 25 uppercase text pages, or one high-resolution page for critical stimuli plus 13 text pages for subject instructions, fixation frames, masks, blank screens, feedback information, and so on. With $32 \mathrm{~KB}$ of RAM, four high-resolution frames and six text frames could be held in memory simultaneously.

Unlike older machines, the color computer needs no hardware modifications for access to the vertical scan synchronization pulse: The hardware connection, to an interrupt-generating input, is already present on the board. A similar connection to the horizontal sync pulse is also provided an can be used to obtain partial-screen displays, effectively increasing the number of available high-resolution frames in memory, by means of frame switching. These inputs may be used for precise timing as well, as discussed below.

While the VDG is built to display text at a density of 16 lines of 32 characters each, the high-resolution format is usable with arbitrary alphabets defined by the user, at up to 24 lines of 43 characters/display. Kitsz (1981) presents an alternative approach, particularly useful to users of unexpanded machines with only a limited amount of memory, using read-only memory character generators to extend the range of characters displayable in text mode. The same article also contains a simple circuit for connecting the color computer to a standard video monitor, bypassing the built-in radio frequency modulator for applications requiring high-definition displays (or in order to use a monitor already in the laboratory.

\section{CASSETTE TAPE-RECORDER INTERFACE}

The Radio Shack color computer is equipped for starting and stopping a cassette tape recorder (or two recorders, one for reading programs or data or sound, and another for recording data) under program control. Programs and data files are read in from and recorded on cassette tape by file name, and unattended operation (for overnight data processing, for example) with program overlays can be readily implemented using closedloop tape cassettes. Sound from cassette can be played back and combined sequentially with sounds from the built-in digital-to-analog (D/A) converter, or from an external source, under program control. This permits highly repeatable sequencing of prerecorded voice instructions and experimental trials in psychological experiments.

\section{ANALOG INPUTS AND OUTPUTS}

The computer comes with a built-in 6-bit (2\% of full-scale resolution) D/A and analog-to-digital (A/D) converter. The output of the $\mathrm{D} / \mathrm{A}$ converter is available at the cassette connector. The "paddle" connectors provide four analog inputs, switchable under software control, to the A/D converter. Existing internal sound connections to the D/A output permit the generation of auditory stimuli with precisely defined waveforms.

\section{DIGITAL I/0}

As stated above, the keyboard appears suitable for response collection in most psychological experiments. Two digital $\mathrm{I} / \mathrm{O}$ lines are available on the "paddle" connectors. If more digital lines are needed, they can be tapped from the keyboard connector up to a total of 15 . Two interrupt inputs, FIRQ (buffered and switchable) and NMI (direct) are available on the cardedge expansion connector. 


\section{TIMING}

The availability of horizontal as well as vertical synchronization pulses at interrupt-generating inputs makes precise timing of video stimuli (Reed, 1979) possible without hardware additions or modifications. An assembly language program to measure reaction times in horizontal scan (HS) units is given in Appendix A. Since each HS unit is equal to $4 / 63 \mathrm{msec}$, a program for unbiased conversion of HS units to milliseconds, such as the one presented in BASIC in Appendix B, must incorporate a "rerandomization" procedure. It can be shown that the increment of reaction time variance due to this procedure is less than $.02 / \mathrm{msec}^{2}$, compared with typical participant-generated reaction time variances of hundreds or thousands of square milliseconds.

\section{NETWORKING}

The color computer incorporates an RS-232 interface for connection to hard-copy devices (printers, teleprinters, plotters) or remote mainframes (through modems or directly). Radio Shack sells networking hardware for color computers, and software for networking at up to 9.6 kbaud is frequently advertised in 80-Microcomputing, Byte, and similar magazines.

\section{EXPANSION}

In laboratories using several "minimal" color computers for experiments, it may be useful to have an "expanded" color computer serve as network controller and software development station. Hardware for simultaneous connection of a printer and a second RS-232 device, such as a network controller, has been advertised in microcomputing magazines. So have packages of soft ware for editing, assembling, and debugging real-time programs for the color computer. With 64-KB randomaccess memory chips, it has become possible to build up to $128 \mathrm{~KB}$ of memory into the machine, although current Radio Shack offerings stop at $32 \mathrm{~KB}$ with $16-\mathrm{KB}$ chips.

The card edge "expansion" connector on the side of the color computer is capable of supporting a wide variety of peripherals. Although now used only for game cartridges, floppy disk systems, and up to $16 \mathrm{~KB}$ of random-access memory outside the SAM's memory management range, the RSCC expansion bus may be expected to permit future connection of auxiliary processors (thanks to a tristating HALT line) and of virtually unlimited amounts of external memory and memory-mapped peripherals (thanks to the SLENB line, which disables internal device selection to free the entire address space for use by external devices).

In terms of software, the RSCC extended BASIC is among the most powerful versions of the language available, including as it does music, graphics, and textformatting capabilities, string-to-numeric and numericto-string conversion capabilities in octal and hexadecimal as well as decimal, built-in editing and renumbering capabilities, and well-designed functions for reserving space for, and interacting with, machine language subprograms. A version of PASCAL for the color computer is being advertised in microcomputer magazines, and it is likely that additional languages, word processors, and so on, will become available with text-display software using the high-resolution video capability. In summary, the Radio Shack color computer appears to be a viable alternative, as a laboratory microcomputer, to other micros costing two or three times as much.

\section{REFERENCES}

KItsz, D. 80 applications. 80 Microcomputing, November 1981, 23, 78-96.

REED, A. V. Microcomputer display timing: Problems and solutions. Behavior Research Methods \& Instrumentation, 1979, 11, $572-576$.

REED, A. V. On choosing an inexpensive microcomputer for the experimental psychology laboratory. Behavior Research Methods \& Instrumentation, 1980, 12, 607-613.

Appendix A

TRSCC Routine for RT in Horizontal Scan Units

$\begin{array}{lllll}\text { RTIMER } & \text { PSHS } & \text { \#FF } & 17 & \text { save everything } \\ \text { LDA } & \# \mathrm{FF} & 2 & \text { make page FF } \\ \text { TFR } & \mathrm{A}, \mathrm{DP} & 6 & \text { the direct page } \\ \text { ORCC } & \# 50 & 3 & \text { mask interrupts and } \\ \text { rep in case interrupted } \\ \text { ORCC } & \# 50 & 3 & \text { open direction } \\ \text { LDA } & \# 38 & 2 & \text { registers for input } \\ \text { STA } & 03, \mathrm{DP} & 4 & \text { and output } \\ \text { STA } & 01, \mathrm{DP} & 4 & \text { set all keyboard } \\ \text { CLB } & & 4 & \text { column bits to input } \\ \text { STB } & 02, \mathrm{DP} & 2 & \text { and row bits } \\ \text { LDA } & \# 7 \mathrm{~F} & 4 & \text { to output } \\ \text { STA } & 00, \mathrm{DP} & 2 & \text { open } \\ \text { LDA } & \text { \#3D } & 4 & \text { data } \\ \text { STA } & 03, \mathrm{DP} & 4 & \text { registers for I/O } \\ \text { STA } & 01, \mathrm{DP} & 4 & \text { output zero on kbd rows } \\ \text { STB } & 00, \mathrm{DP} & & \end{array}$




\begin{tabular}{|c|c|c|c|c|}
\hline & LDA & $\# 34$ & 2 & disable \\
\hline & STA & 21,DP & 4 & all other \\
\hline & STA & $23, \mathrm{DP}$ & 4 & interrupts \\
\hline & LDX & $\# 0000$ & 3 & preset registers \\
\hline & LEAU & $\mathrm{X}$ & 4 & for counting HS pulses \\
\hline & LDB & PRESETLO & 2 & and limit to preset \\
\hline & LDY & PRESETHI & 4 & number of video frames \\
\hline & LDA & $00, \mathrm{DP}$ & 4 & clear interrupt flags \\
\hline & LDA & $02, \mathrm{DP}$ & 4 & initialized in 96 cycles \\
\hline \multirow[t]{4}{*}{ SYNCH } & SYNC & & $4+$ & wait for interrupt \\
\hline & LEAX & $\# 01, \mathrm{X}$ & 5 & increment low 16 bits \\
\hline & $\mathrm{BNE}$ & TIMECHK & 3 & and if zero reached \\
\hline & LEAU & $\# 01, \mathrm{U}$ & 5 & also hi 16 of HS count \\
\hline \multirow[t]{6}{*}{ TIMECHK } & LDA & $03, \mathrm{DP}$ & 4 & check for VS interrupt \\
\hline & BPL & RESETINTS & 3 & and if flagged then \\
\hline & CLA & & 2 & the low 8 bits of VS \\
\hline & ADDD & $\$ 0001$ & 4 & are incremented and the \\
\hline & LEAY & A,Y & 5 & count extended to hi 16 \\
\hline & BEQ & TIMEOUT & 3 & to check for timeout \\
\hline \multirow[t]{4}{*}{ RESETINTS } & LDA & $00, \mathrm{DP}$ & 4 & otherwise clear HS flag \\
\hline & LDA & $02, \mathrm{DP}$ & 4 & and VS; check keyboard \\
\hline & COMA & & 2 & go back if not pressed \\
\hline & BEQ & SYNCH & 3 & loop is $51+$ cycles long \\
\hline \multirow[t]{3}{*}{ PRESSED } & STU & RT3,PCR & 6 & pressed: store hi $16 \&$ \\
\hline & STX & RT1,PCR & 6 & low 16 bits of HS count \\
\hline & BRA & FINISH & 3 & then finish up \\
\hline \multirow[t]{2}{*}{ TIMEOUT } & STY & RT3,PCR & 7 & if timed out put zeros \\
\hline & STY & RT1,PCR & 7 & in place of HS count \\
\hline \multirow[t]{7}{*}{ FINISH } & LDA & $\# 3 \mathrm{C}$ & 2 & to finish disable both \\
\hline & STA & $03, \mathrm{DP}$ & 4 & VS and \\
\hline & STA & $01, \mathrm{DP}$ & 4 & HS interrupts \\
\hline & LDA & $00, \mathrm{DP}$ & 4 & and clear \\
\hline & LDA & $02, \mathrm{DP}$ & 4 & both interrupt flags \\
\hline & PULS & $\# \mathrm{FF}$ & 17 & restore old context \\
\hline & RTS & & 5 & and return \\
\hline RT3-RT0 & DATA & 4 BYTES & & \\
\hline
\end{tabular}

Appendix B

Horizontal Scan Units to Milliseconds BASIC Conversion Routine

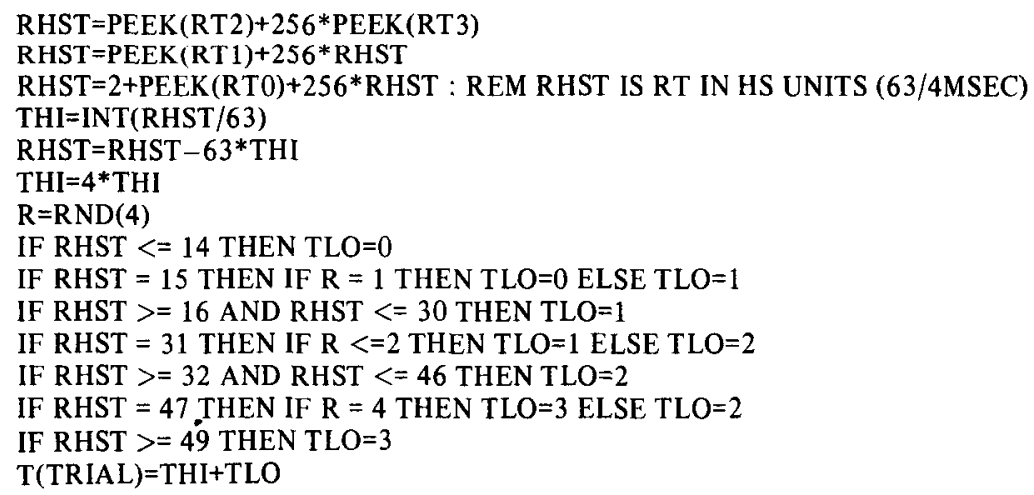

